\title{
Permeance based modelling of the core corners considering magnetic material non- linearity
}

\author{
M. Luo and D. Dujić
}

This material is posted here with permission of the IEEE. Such permission of the IEEE does not in any way imply IEEE endorsement of any of EPFL's products or services. Internal or personal use of this material is permitted. However, permission to reprint / republish this material for advertising or promotional purposes or for creating new collective works for resale or redistribution must be obtained from the IEEE by writing to pubs-permissions@ieee. org. By choosing to view this document, you agree to all provisions of the copyright laws protecting it. 


\title{
Permeance Based Modelling of the Core Corners Considering Magnetic Material Nonlinearity
}

\author{
Min Luo, Dražen Dujić \\ École Polytechnique Fédérale de Lausanne - EPFL \\ Power Electronics Laboratory - PEL \\ Station 11, CH-1015, Lausanne, Switzerland \\ min.luo@epfl.ch,drazen.dujic@epfl.ch
}

\begin{abstract}
Lumped magnetic circuit provides possibility for fast transient simulation of magnetic component together with power electronics circuit. The simplified geometry representation however is not able to completely reflect the real field distribution, especially when material nonlinearity is included. To model electrical characteristic of the magnetic components closer to the reality, special treatment is needed to determine geometrical parameters of the lumped representation. In this paper, modeling of the curved areas (e.g. core corners) based on permeances is presented and compared with FEM simulations.
\end{abstract}

Index items-magnetic modeling, permeance, saturation

\section{INTRODUCTION}

Inductive components usually use magnetic core material to guide magnetic flux. Finite-element-method (FEM) is able to represent the core geometry and solve the field distribution accurately. However, this type of model is computationally expensive if incorporated into circuit simulation. In comparison, lumped magnetic circuit approach is able to achieve high simulation speed, for which reluctance- and permeance representation are available today. The permeance representation proposed in [1] can be solved by ordinary differential equation (ODE) solver without any time-consuming iterations, and is therefore preferred for system-level power electronic simulations [2]. However, the simplification of core geometry required by the lumped modeling approach may result in considerable error, if the material's nonlinearity should be considered.

In lumped magnetic circuit, complex core geometry can be split into segments, where each segment represents a certain part of the core. Typical example is the three-leg transformer core with parallel connection of reluctances [3]. The reluctance values were determined by fitting the data from the externally measured electrical characteristics (e.g. opencircuit and short-circuit test). Work of [4] and [5] transformed the magnetic circuit back to electrical equivalent circuit. In these publications however, the direct relationship between the lumped component value (permeance or reluctance) and the core-geometry parameters was not utilised.

To make use of this relationship, single segment is characterised by three parameters, namely the geometry-related cross section area $A$ and magnetic path length $l$, as well as material-related permeability $\mu$. Geometry parameters can

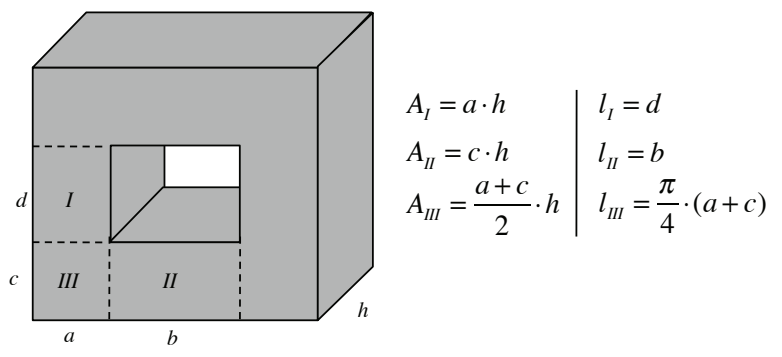

Fig. 1. Simplified geometry equivalent of the magnetic core

be directly achieved by measuring the core dimension, while material parameter are obtained from the data-sheet. These three parameters will be transformed into a reluctance or permeance value during simulation, as shown in [6] for a three leg transformer core. In this case, unique cross-section area and magnetic path length was assigned to both corner and straight limb of one core leg. In reality however, the corner and straight limbs may have different equivalent cross-section.

Authors of [7] defined the geometry parameters of the straight limbs and corner separately. Plenty of works of others [8], [9] and [10] followed this definition. The crosssection area and magnetic path length of the straight limb and corner were calculated as shown in Fig. 1. Due to relatively homogeneous field distribution in the straight limb (volume I and II), the cross-section and length of the cuboid were directly taken. For the corner (volume III), the geometry was regarded as one quarter of a toroidal core with small inner radius, considering the fact that the sharp corner area is usually poorly utilised. The mid-line of the toroidal quarter was simply chosen as the equivalent path length, as depicted in Fig. 2. The overall accuracy of the core model using this mid-line approximation for corner should be sufficient, if the magnetic path length of the straight limbs are significantly longer than that of the corner's, so that the corner does not dominate the dynamic characteristic. However if this is not the case, the fidelity of the mid-line representation might be erroneous due to the lack of analytical derivation.

A polynomial formulae for the corner geometry, derived from field analysis in [11], was used in [12] for a rotary transformer core. This formulae was only valid for linear material. If the core material is non-linear, the permeability 


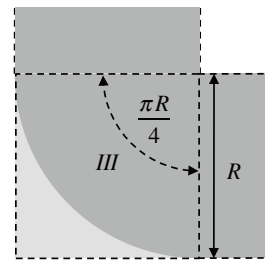

Fig. 2. Simplified geometry equivalent of the core corner

$\mu$ along the radius direction in Fig. 2 are different due to different local $H$, such that no explicit form can be achieved. In this paper a new approach for the core-corner modeling is presented, which is also valid under core material nonlinearity.

\section{ANALYSiS}

Taking a toroidal core as an example, the inner and outer radius are $R_{\text {in }}$ and $R_{\text {out }}$ respectively, while the thickness in axial direction is $D$. In addition, let assume that a winding with number of turns $N$ and current $I$ is installed. Due to symmetry, the field strength $H$ is homogeneous along tangential direction, while along radius direction is defined as:

$$
H(r)=\frac{N I}{2 \pi r}=\frac{F}{2 \pi r}
$$

where $F$ is the magneto-motive force (MMF). If the core material is linear and has constant relative permeability $\mu_{r}$, the flux density $B$ can be directly calculated as:

$$
B=\mu_{r} \mu_{0} \frac{F}{2 \pi r}
$$

The flux $\Phi$ inside the core is given as surface integration of $B$ on the cross-section area:

$$
\Phi=D \cdot \int_{R_{\text {in }}}^{R_{\text {out }}} \mu_{r} \mu_{0} \frac{F}{2 \pi r} d r=\frac{\mu_{r} \mu_{0} D F}{2 \pi} \ln \left(\frac{R_{\text {out }}}{R_{\text {in }}}\right)
$$

In order to translate the relationship into permeance representation, the flux's derivation by $\mathrm{F}$ is calculated:

$$
\mathscr{P}=\frac{d \Phi}{d F}=\mu_{r} \mu_{0} \frac{D\left(R_{\text {out }}-R_{\text {in }}\right)}{2 \pi\left(R_{\text {out }}-R_{\text {in }}\right) / \ln \left(R_{\text {out }} / R_{\text {in }}\right)}
$$

where the numerator $D\left(R_{\text {out }}-R_{\text {in }}\right)$ is equal to the core's equivalent cross-section area $A_{e q}$, while the denominator $2 \pi\left(R_{\text {out }}-R_{\text {in }}\right) / \ln \left(R_{\text {out }} / R_{\text {in }}\right)$ corresponds to the length of the equivalent magnetic path $l_{e q}$.

When the nonlinear effect of the core material is taken into account, one has to pay attention as the previous relationship is no longer valid. If reversible magnetization is assumed, $B$ should be single valued nonlinear function of $H$. The permeance becomes:

$$
\mathscr{P}=\frac{d \Phi}{d F}=D\left(\int_{R_{\text {in }}}^{R_{\text {out }}} \frac{\partial B(H)}{\partial F} d r\right)
$$

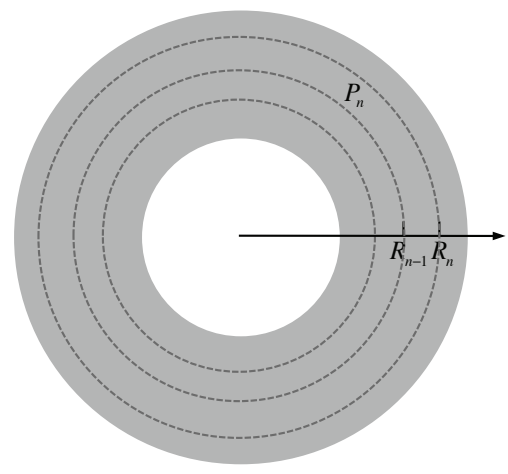

Fig. 3. Toroidal core split into multiple layers

Since the field strength $H$ still follows (1), the equation can be rewritten as:

$$
\mathscr{P}=D \cdot \int_{R_{\text {in }}}^{R_{\text {out }}} \mu_{0} \mu_{r}\left(\frac{F}{2 \pi r}\right) \frac{1}{2 \pi r} d r
$$

It is important to understand that $\mu_{r}$ in (6) is the differential permeability, and is equal to the local slope on the B-H curve at given $H(d B / d H$ instead of $B / H)$, normalized by $\mu_{0}$. The equation (6) indicate that the material has different saturation level along the radius direction, and it is not possible to extract an explicit equivalent length directly as done in (4).

In order to represent the nonlinearity with acceptable accuracy using lumped permeance, one possible way is to split the core into multiple layers as shown in Fig. 3. The total permeance can be written as:

$$
\mathscr{P}=\sum_{n=1}^{N_{l}} \mathscr{P}_{n}=\sum_{n=1}^{N_{l}} D \cdot \int_{R_{n-1}}^{R_{n}} \mu_{0} \mu_{r}\left(\frac{F}{2 \pi r}\right) \frac{1}{2 \pi r} d r
$$

where $R_{n}-1$ and $R_{n}$ t are inner- and outer radius of each layer, respectively. Please note that split is defined in such a way so that $R_{n}-R_{n-1}$ are the same for all layers. The differential permeability $\mu_{r}$ is assumed to be unique in each layer, therefore it can be moved outside of the integration. The permeance value of single layer thus becomes:

$$
\mathscr{P}_{n}=\mu_{0} \mu_{r}\left(\frac{F}{l_{e q, n}}\right) \frac{D\left(R_{n}-R_{n-1}\right)}{l_{e q, n}}
$$

and the equivalent magnetic path length $l_{e q}$ of each layer has the same format as (4)

$$
\left.l_{e q, n}=2 \pi\left(R_{n}-R_{n-1}\right) / \ln \left(R_{n} / R_{n-1}\right)\right)
$$

The finer the core is split, the better accuracy can be achieved, at the expense of additional computational effort. This approach is equivalent to connection of $n$ permeances in parallel, as shown in Fig. 4. 


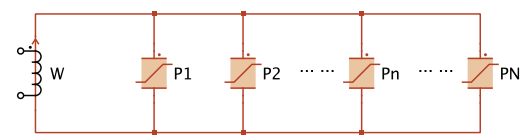

Fig. 4. Equivalent magnetic circuit of the toroidal core when split into multiple layers

\section{EvaluATION}

Simulations have been carried out to evaluate proposed approach. At, firstly the $B-H$ characteristic of a certain magnetic material (2605SA1) is modelled using atan fit [13], as shown in Fig. 5 and three areas of interest are defined.

\section{A. Toroidal core}

For the simulation, a toroidal core with inner radius $R_{\text {in }}=$ $2 \mathrm{~mm}$, outer radius $R_{\text {out }}=13 \mathrm{~mm}$ and thickness $D=11 \mathrm{~mm}$ is used. At first the model is created with lumped-permanences in system-level power electronics simulation platform PLECS, in circuit configuration as shown in Fig. 6. Note that only one permeance is present with value equal to multiple permeances in parallel connection, thus avoiding increased number of state variables. The AC voltage source with $200 \mathrm{~Hz}$ frequency and 90 degree phase shift is applied directly to the winding with turns number $N=5$, to avoid initial DC-bias of the flux.

2D-FEM model that accurately represents the selected core geometry is established in COMSOL for verification reasons. The conductivity of the core is set to $\sigma=0 S / m$, such that the eddy current effect is excluded (Fig. 7).

Three operating conditions characterized with different saturation rates are configured for the time-domain simulation. In the first case the core operates in unsaturated region, which is the part of the $\mathrm{BH}$ curve near $H=0$ (as marked in Fig. 5). The amplitude of the AC voltage is set to $0.3 \mathrm{~V}$.

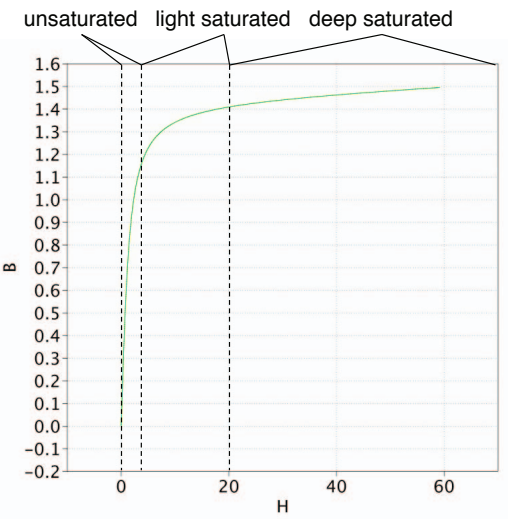

Fig. 5. Modelled B-H curve of alloy 2605SA1 with three areas of interest

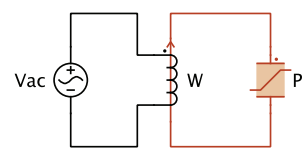

Fig. 6. Circuit configuration of the toroidal core simulation model

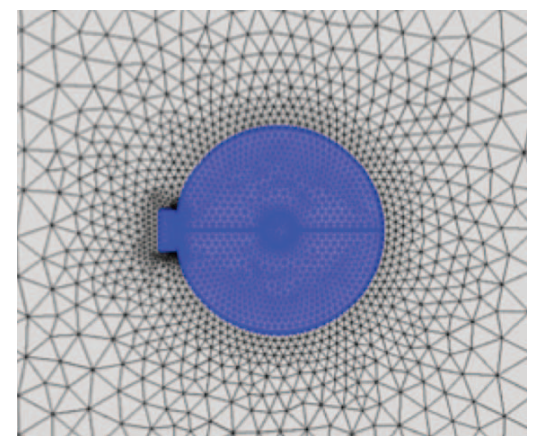

Fig. 7. Meshed FEM model of the toroidal core

Fig. 8 shows three current waveform, corresponding to the new layer-split representation (noted as "layer"in legend), mid-line representation by [7] (noted as "linear" in legend) and FEM (noted as "FEM" in legend) respectively. For the unsaturated core, the result show that layer-split modeling provides results much closer to FEM simulations, in contrast to the mid-line representation. For the core operated in deep saturation with an $\mathrm{AC}$ voltage amplitude of $4 \mathrm{~V}$, results in Fig. 9 show even larger error between the mid-line representation and FEM. In comparison, the layer-split representation still matches FEM well.

When the core operates in light saturation area, where the B$\mathrm{H}$ curve has largest curvature, both the mid-line and layer-split representation match the FEM result well (Fig. 10). To explore the reason, the field distribution from the FEM simulation is analysed, as Fig. 11 shows. The $H$ and $B$ distribution along the radius direction from FEM simulation as well as theoretical calculation from section II are depicted in Fig. 12 and Fig. 13, and they show excellent agreement.

$H$ distribution in Fig. 12 is a nonlinear and follows the equation (1), while $B$ distribution becomes almost linear along the radius direction, due to the convex B-H characteristic (Fig. 13). Therefore the flux can be approximated as:

$$
\Phi \approx D\left(R_{\text {out }}-R_{\text {in }}\right) \frac{B\left(R_{\text {in }}\right)+B\left(R_{\text {out }}\right)}{2}
$$

Derivation by $d F$, yields the permeance:

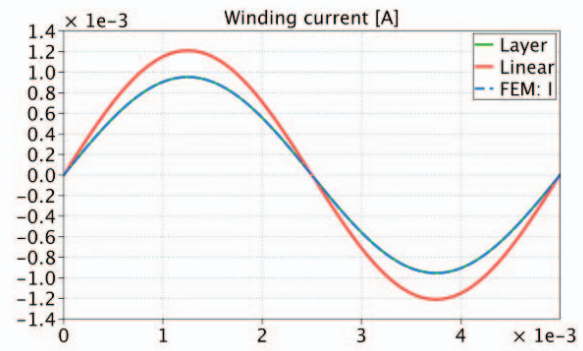

Fig. 8. Simulated winding current of the toroidal core - unsaturated 


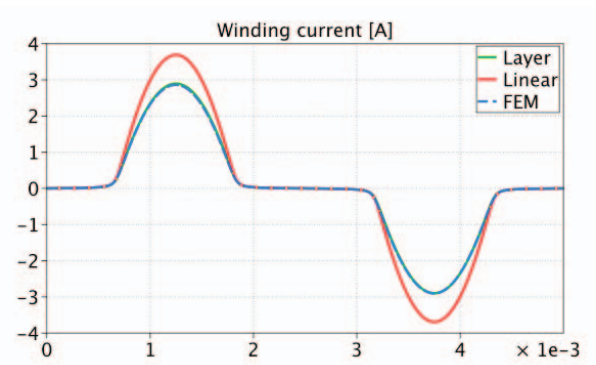

Fig. 9. Simulated winding current of the toroidal core - deeply saturated

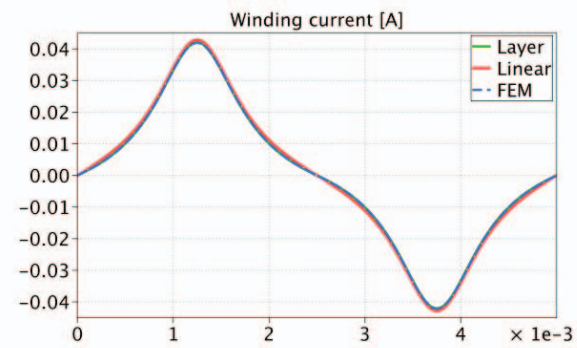

Fig. 10. Simulated winding current of the toroidal core - lightly saturated

$$
\begin{aligned}
& \frac{d \Phi}{d F}=\frac{B_{\text {sat }} D\left(R_{\text {out }}-R_{\text {in }}\right)}{\pi a} \\
& \cdot\left(\frac{1}{2 \pi R_{\text {out }}+\frac{F^{2}}{R_{\text {out }} \cdot 2 \pi a^{2}}}+\frac{1}{2 \pi R_{\text {in }}+\frac{F^{2}}{R_{\text {in }} \cdot 2 \pi a^{2}}}\right)
\end{aligned}
$$

Considering the fact that in the light saturation area, the following relationship is usually fulfilled:

$$
2 \pi R_{\text {out }} \ll \frac{F^{2}}{R_{\text {out }} \cdot 2 \pi a^{2}}
$$

the term $2 \pi R_{\text {out }}$ can be neglected and $\mathscr{P}$ becomes:

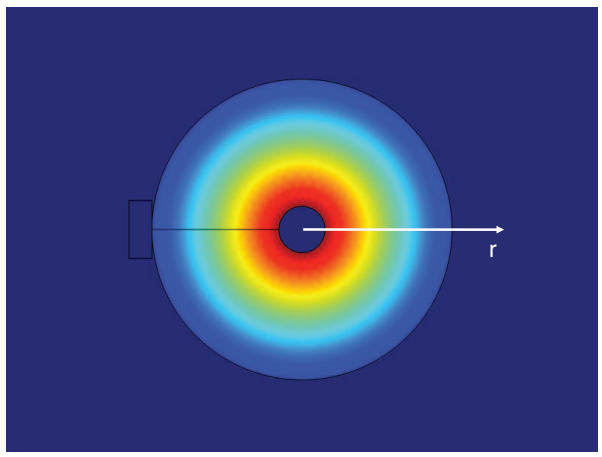

Fig. 11. FEM flux density at current peak in light saturation

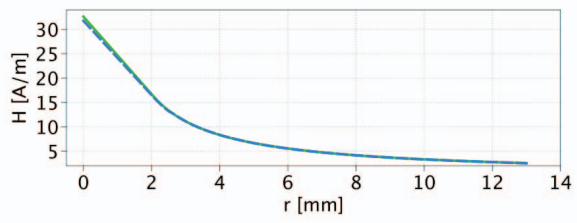

Fig. 12. $\mathrm{H}$ along radius direction at current peak in light saturation

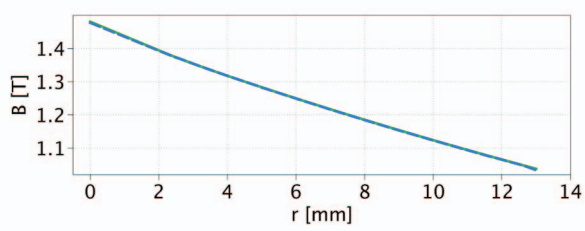

Fig. 13. B along radius direction at current peak in light saturation

$$
\begin{aligned}
\mathscr{P}=\frac{d \Phi}{d F}= & \frac{D\left(R_{\text {out }}-R_{\text {in }}\right) B_{\text {sat }}}{\pi a} \\
& \cdot\left(\frac{1}{\frac{F^{2}}{R_{\text {out }} \cdot 2 \pi a^{2}}}+\frac{1}{\frac{F^{2}}{R_{\text {in }} \cdot 2 \pi a^{2}}}\right)
\end{aligned}
$$

This can be further reduced to:

$$
\mathscr{P}=\frac{2 B_{\text {sat }} a D\left(R_{\text {out }}-R_{\text {in }}\right)\left(R_{\text {out }}+R_{\text {in }}\right)}{F^{2}}
$$

On the other hand, if the mid-line representation for the magnetic path length is applied, the approximated permeance is given as:

$$
\begin{aligned}
\mathscr{P}_{\text {midline }}=\frac{d \Phi}{d F}= & \frac{B_{\text {sat }} D\left(R_{\text {out }}-R_{\text {in }}\right)}{\pi a} \\
& \cdot \frac{1}{2 \pi \frac{R_{\text {out }}+R_{\text {in }}}{2}+\frac{F^{2}}{\frac{R_{\text {out }}+R_{\text {in }}}{2} \cdot 2 \pi a^{2}}}
\end{aligned}
$$

The same simplification is valid here as well, therefore:

$$
2 \pi \frac{R_{\text {out }}+R_{\text {in }}}{2} \ll \frac{F^{2}}{\frac{R_{\text {out }}+R_{\text {in }}}{2} \cdot 2 \pi a^{2}}
$$

and $\mathscr{P}_{\text {midline }}$ becomes:

$$
\mathscr{P}_{\text {midline }}=\frac{2 B_{\text {sat }} a D\left(R_{\text {out }}-R_{\text {in }}\right)\left(R_{\text {out }}+R_{\text {in }}\right)}{F^{2}}
$$

Finally, $\mathscr{P}_{\text {midline }}$ turns out to be equal to $\mathscr{P}$, which can explain the small error of the mid-line representation, when simulating the light saturation condition. However one should keep in mind that, this situation is just a coincidence that happens in certain operation condition. Regarding the simulation speed, the magnetic circuit model takes maximum 0.6 seconds while FEM more than 60 seconds on the same computer. 


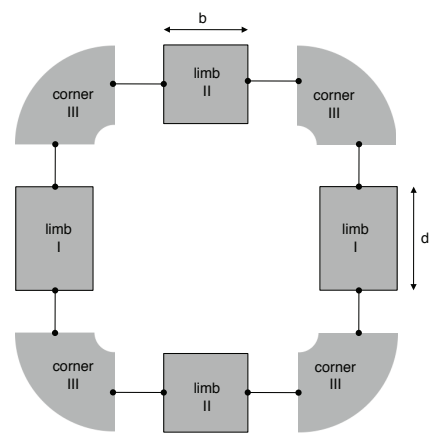

Fig. 14. U-core extended from toroidal core

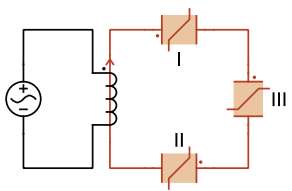

Fig. 15. Lumped model of the U-core

\section{B. U-shape core}

The toroidal core from the last evaluation case is extended to a U-shape core by inserting straight limbs, as illustrated in Fig. 14. U-cores with the straight limbs of different lengths are modeled in both lumped way and using FEM. The straight limb's geometric parameters are configured following the equations in Fig. 1. The magnetic path lengths are $b=13 \mathrm{~mm}$, $d=13$ (referred as "short" afterwards) and $b=13 \mathrm{~mm}$, $d=50 \mathrm{~mm}$ (referred as "long" afterwards), respectively.

The lumped model is established as series connection of three blocks, representing the parts "I", "II" and "III" in Fig. 1 (note that the parallel limb pair of "I" and "II" as well as the four corners "III" are merged into one respectively, as shown in Fig. 15).

The time domain simulation results of winding current are shown in Fig. 16, Fig. 17 and Fig. 18 for three different saturation conditions. Comparing the simulation result of the short and long U-core in different saturation conditions, it can be found that the difference between the mid-line and layer-split representation decrease as the straight limbs' length increases.

For the layer-split approach, the error of the current peak compared to the FEM result are evaluated against number of layers, as the coloured curves displayed in Fig. 19, Fig. 20. The percentage values enclosed by brackets in the legends are the errors from mid-line approach, compared to FEM. In unsaturated and deep saturated operation, the errors of the layer-split approach change only slightly with increasing number of layers, which are significantly lower, compared to the mid-line approach (Fig. 19, Fig. 20). In lightly saturated operation, the errors of layer-split approach do not show significant improvement compared with mid-line approach, due to the reasons discussed earlier. In this case, the errors of layer-split decrease nearly exponentially with the increased
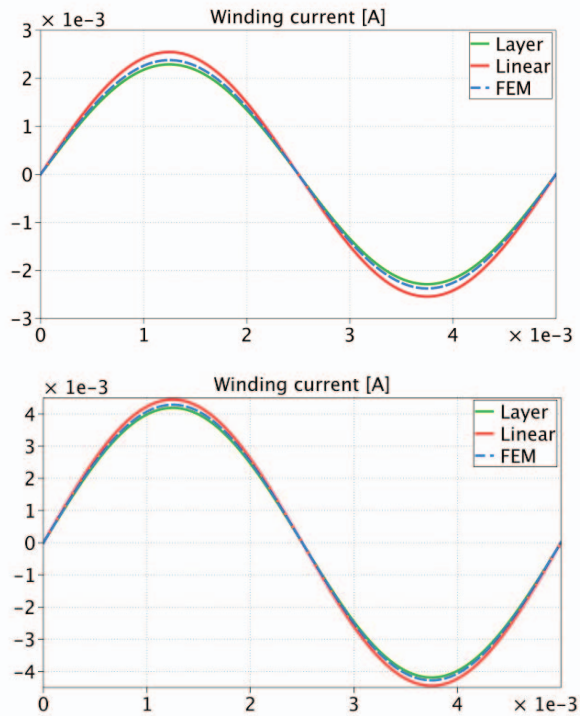

Fig. 16. Simulated winding current of unsaturated short and long U-core
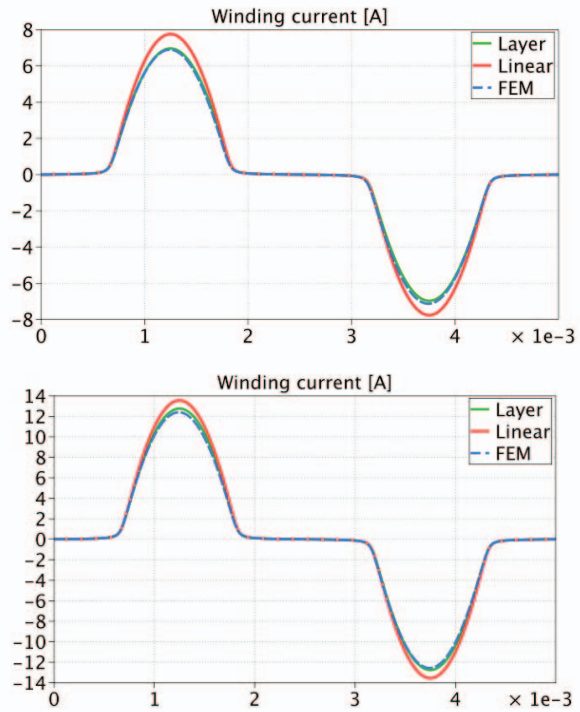

Fig. 17. Simulated winding current of deeply saturated short and long U-core

number of layers.

Based on this, three layers can be chosen as a generally acceptable configuration, which is able to deliver reasonable error $(\leq 4 \%)$ in wide operation range. Higher layer count can be chosen, if further reduction of error in light saturation is desired. With $N_{l}=20$ the current peak error of the three geometries in light saturated saturation are $0.2 \%, 0.6 \%$ and $0.4 \%$ respectively. For unsaturation and deep saturation however, increasing layer count over 3 does not bring significant improvement. In comparison, the mid-line approach is only coincidentally suitable for light saturation, and will result in large error in unsaturation and deep saturation. 

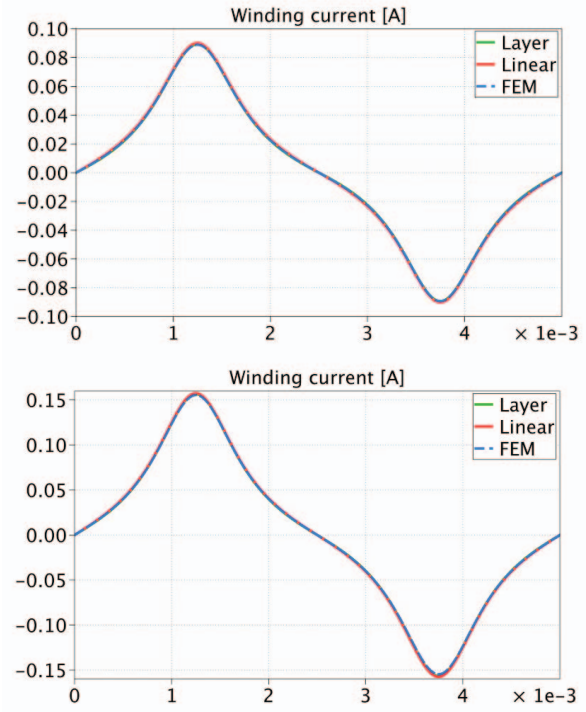

Fig. 18. Simulated winding current of lightly saturated short and long U-core

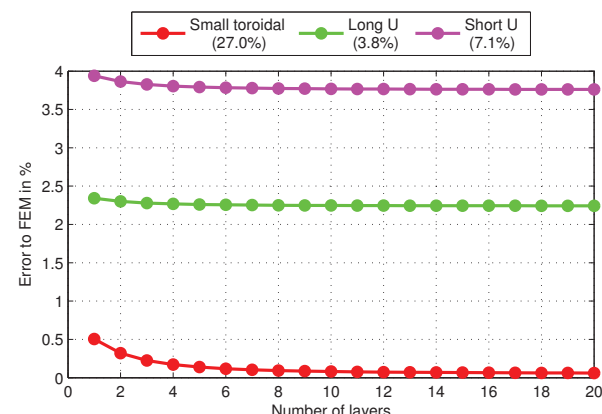

Fig. 19. Peak current error of layer-split versus FEM - unsaturated

\section{CONCLusion}

Permeance based layer-split approach to model a core corner for system level time-domain simulation for transformer or inductor, is proposed and compared with the conventional "mid-line" method and the FEM field simulation in terms of accuracy, using current peak error as figure of merit. The evaluation result show that the layer-split approach is able to provide better accuracy in wide operation range, especially when the dimension of the corner dominates the core geometry. Uniform core split in multiple layers has been considered, while other distributions are possible as well. Further effort will be invested to extend this approach to cores with nonsquare cross section and hysteresis material characteristic.

\section{REFERENCES}

[1] D. Hamill, "Lumped equivalent circuits of magnetic components: the gyrator-capacitor approach," in IEEE Transactions on Power Electronics, vol. 8, 1994, pp. 97-103.

[2] J. S. J. Allmeling, W. Hammer, "Transient simulation of magnetic circuits using the permeance-capacitance analogy," in Control and Modeling for Power Electronics (COMPEL), IEEE 13th Workshop on, 2012.

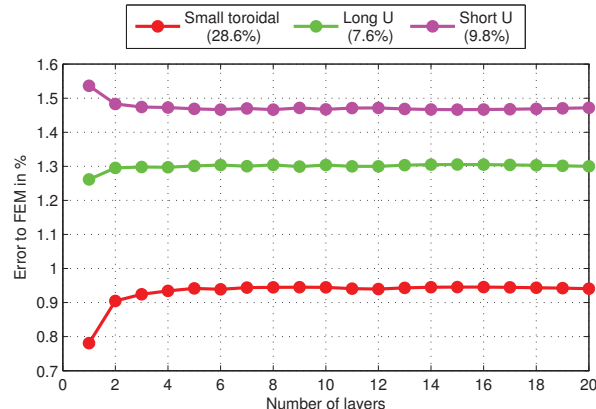

Fig. 20. Peak current error of layer-split versus FEM - deeply saturated

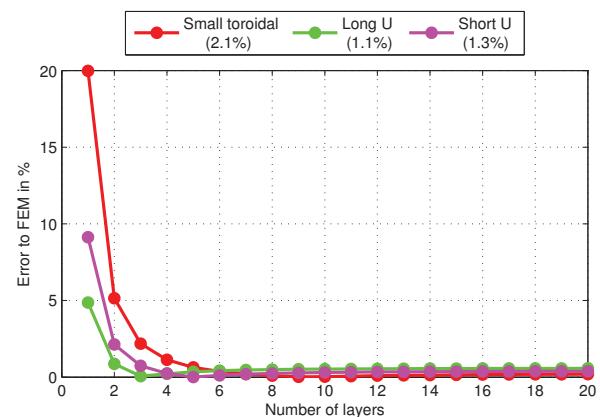

Fig. 21. Peak current error of layer-split versus FEM - lightly saturated

[3] D. J. R. E. F. Fuchs, Y. You, "Modelling and simulation and their validation of three-phase transformers with three legs under dc bias,' in IEEE Transactions on Power Delivery, vol. 14, no. 2, 1999, pp. 443 449.

[4] S. S. V. X. Chen, "Three-phase three-winding core-type transformer model for low-frequency transient studies," in IEEE Transactions on Power Delivery, vol. 12, no. 2, 1997, pp. 775-782.

[5] B. A. Mork, "Five-legged wound-core transformer model: derivation, parameters, implementation and evaluation," in IEEE Transactions on Power Delivery, vol. 14, no. 4, 1999, pp. 1519-1526.

[6] M. P. M. Elleuch, "A contribution to the modelling of three phase transformers using reluctances," in IEEE Transactions on Magnetics, vol. 32, no. 2, 1996, pp. 335-343.

[7] E. C. Snelling, Soft Ferrite: Properties and Applications. London Iliffe Books Ltd., 1969.

[8] F. K. J. W. K. C. Marxgut, J. Muehlethaler, "Multiobjective optimization of ultraflat magnetic components with pcb-integrated core," in IEEE Transactions on Power Electronics, vol. 28, no. 7, 2013, pp. 3591-3602.

[9] J. Mühlenthaler, "Modelling and multi-objective optimizaiton of inductive power components," Ph.D. dissertation, ETH Zürich, 2012.

[10] J. C. J. C. O.-G. I. Hernandez, F. de Leon, "Modelling transformer cores joints using gaussian models for the magnetic flux density and permeability," in IET Electric Power Applications, vol. 4, 2010, pp. 761771.

[11] G. M. Stein, "Influence of the core form upon the iron losses of the transformer," in AIEE Transaction, vol. 67, 1948, pp. 95-105.

[12] P. G. B. J. van Vlerken, "Lumped modelling of rotary transformers, heads and electronics for helical-scan recording," in IEEE Transactions on Magnetics, vol. 31, no. 2, 1995, pp. 1050-1055.

[13] C. Perez-Rojas, "Fitting saturation and hysteresis via arctangent funcitons," in IEEE Power Engineering Review, vol. 20, no. 1, 2000, pp. $55-57$. 